\title{
Neonatal lenticulostriate vasculopathy: further characterisation
}

\author{
I R Makhoul, I Eisenstein, P Sujov, M Soudack, T Smolkin, A Tamir, M Epelman
}

See end of article for authors' affiliations

.....................

Correspondence to: Dr Makhoul, Department of Neonatology, Rambam Medical Center, Bat-Galim, Haifa 31096, Israel: makhoul@ rambam.health.gov.il

Accepted 30 October 2002

\begin{abstract}
Background: Lenticulostriate vasculopathy (LSV) is sometimes detected on routine brain ultrasonography in neonates, and is often associated with various perinatal and neonatal abnormalities. However, most reports on LSV are retrospective with no controls.

Objectives: To compare the perinatal and neonatal clinical characteristics of neonates with LSV with matched controls and to summarise all published reports of LSV.

Design: A prospective study that summarises the clinical, laboratory, and neurosonographic data of neonates with LSV.

Methods: Of 1184 neonates admitted to the neonatal intensive care unit (NICU) during a three year period, 857 had a routine head ultrasound examination. Twenty one had LSV, and were compared with 42 matched controls with regard to gestational, perinatal, neonatal, laboratory, and neurosonographic characteristics.

Results: LSV was detected in 21 of the 857 (2.45\%) neonates. It was bilateral in 10 of the 21 cases and located in the thalamus $(n=14)$ and basal ganglia $(n=7)$. Infants with LSV were not significantly different from matched controls in most tested variables. However, compared with the control group, the LSV group included significantly more multiple births and more disturbances in amniotic fluid volume, but less meconial amniotic fluid. In addition, the patients with LSV required fewer blood transfusions and less phototherapy.

Conclusions: Except for more multiple births, neonates with LSV did not display more adverse findings than their matched controls.
\end{abstract}

enticulostriate vasculopathy (LSV) is detected in $0.4 \%$ of all liveborn neonates ${ }^{1}$ and $1.9-5.8 \%$ of ill neonates, ${ }^{2-7}$ using -cranial ultrasound (US) as hyperechogenic arteries in the thalamus and basal ganglia.

LSV can be either unilateral or bilateral, with a branching, linear (strip-like), or punctate shaped pattern. ${ }^{1458}$ Histopathological examination shows that most lenticulostriate arteries involved in LSV are medium sized, have thickened and hypercellular walls without fibrosis or hyalinisation, but with intramural and perivascular deposition of amorphous basophilic material, iron, and calcium, ${ }^{45}$ and signs of vessel wall damage. ${ }^{4}$

Since the initial description of LSV by Grant $e t ~ a l^{10}$ in 1985 , more than 25 reports of neonatal LSV have been published, in which LSV appeared to be associated with a variety of congenital and acquired neonatal conditions, such as fetal and neonatal infections, mainly cytomegalovirus, ${ }^{1-4}$ 6-9 $^{11-15}$ chromosomal aberrations, ${ }^{2-46891617}$ hypoxic/ischaemic conditions, ${ }^{4-6}$ congenital heart disease, ${ }^{4}$ fetal alcohol or drug exposure, ${ }^{3-6}$ congenital malformations, ${ }^{146}$ neonatal lupus erythematosus, ${ }^{36}$ twin to twin transfusion, ${ }^{18}$ sialidosis, ${ }^{19}$ hydrops fetalis, ${ }^{6}$ and diabetic fetopathy. ${ }^{1}$

Most published reports of LSV are either of large retrospective studies ${ }^{2-7}$ or are case reports, ${ }^{10-24}$ except for one prospective study by Kriss et al $^{8}$ in which prospective screening of brain sonograms performed in the neonatal intensive care unit (NICU), rather than of neonates, was conducted. From the literature on LSV, no clear conclusions can be drawn about the consequences of LSV on the sick neonate. Therefore we performed a prospective study with the following aims: (a) to summarise the clinical, laboratory, and neurosonographic data of all neonates with LSV in our NICU; $(b)$ to compare the perinatal and neonatal clinical characteristics of neonates with LSV with those of matched controls; $(c)$ to elucidate possible meaningful clinical trends from our observations. We expected to discern characteristics that would delineate the relevance of a finding of LSV in the sick neonate.

\section{METHODS}

\section{Study design and groups}

This prospective study was conducted between l February 1999 and 31 January 2002 in the tertiary centre NICU at Rambam Medical Center. During the study period, 1184 neonates were admitted; 857 of them had a routine head US examination. The remaining 327 included neonates transferred to our NICU for surgical interventions, infants whose stay in the NICU was short term (transient tachypnoea of newborn, polycythaemia, jaundice), and some extremely low birthweight infants who died within 48 hours of admission.

Of the 857 neonates who had head US examinations, LSV was detected in 21 (2.45\%); these comprised the study group. Each patient in the study group was matched with the two subsequent neonates born at the same gestational age $( \pm 3$ days) and in the NICU, but who did not have LSV (control group; $n=42$ ). Neonates of both groups were born after 27-41 weeks gestation. When LSV occurred in one or both twin neonates, they were matched to control twin neonates, observing the same order of twinning (first with first, second with second).

\section{Data collection}

In both the study and control group, data were prospectively collected on the neurosonographic characteristics of LSV (table 1), maternal, gestational, and perinatal characteristics

Abbreviations: LSV, lenticulostriate vasculopathy; NICU, neonatal intensive care unit; US, ultrasonography; TORCH, toxoplasma, other viruses, rubella, cytomegalovirus, herpes virus 


\begin{tabular}{ll}
$\begin{array}{l}\text { Table } 1 \text { Sonographic characteristics of neonates } \\
\text { with LSV ( } \mathrm{n}=2 \text { 1) }\end{array}$ & \\
\hline Variable & Number \\
\hline NICU admissions during the study period & 1184 \\
Neonates with head sonograms & $857(72.4 \%)$ \\
Number of neonates with LSV & $21(2.45 \%)$ \\
LSV diagnosed on 1 st US & 19 \\
LSV diagnosed on 2nd US & 2 \\
Age at examination (days) 1st US & $8.8(5.4)$ \\
LSV & 11 \\
Unilateral & 10 \\
Bilateral & 14 \\
Location of LSV & 7 \\
Thalamus & \\
Basal ganglia & 3 \\
IVH & 2 \\
$\quad$ Grade 1-2 & 2 \\
Grade 3-4 & 0 \\
Ventriculomegaly & \\
Hydrocephalus &
\end{tabular}

LSV, Lenticulostriate vasculopathy; NICU, neonatal intensive care unit; IVH, intraventricular haemorrhage.

(table 2), and neonatal variables and medical interventions (table 3). In addition, we conducted a comprehensive review of the literature on neonatal LSV (table 4). We could not perform a long term neurodevelopmental follow up in our series because of lack of data for most patients.

\section{Head US examination}

A first head US examination was performed at 8.2 (5.4) days and 8.6 (6.1) days after birth in the study and control groups respectively (mean (SD)). Scans were performed with a $5 \mathrm{MHz}$ convex transducer (SSD-1400; Aloka Co, Tokyo, Japan) and a 5-8 MHz curved transducer (HDI 5000; Advanced Technology Laboratories, Bothell, Washington, USA). Each examination included coronal and bilateral parasagittal views of the brain. Colour Doppler examination was performed in three cases.

\section{Statistical analysis}

For comparison of the results of the study and control groups, we used Student's $t$ test for continuous variables (duration of mechanical ventilation), the Wilcoxon test for ordinal variables (Apgar score), and the $\chi^{2}$ test for dichotomic variables. $p<0.05$ was considered significant.

\section{RESULTS}

\section{Incidence and sonographic characteristics of LSV}

Table 1 shows that the incidence of LSV in our study was $2.45 \%$ of NICU admissions. The gestational age distribution of the 21 patients with LSV was as follows: < 30 weeks, one; 30-33 weeks, six; 34-37 weeks, eight; > 37 weeks, six. LSV was mostly of linear branching pattern (fig $1 \mathrm{~A}, \mathrm{~B}$ ) and confirmed to be vascular by colour Doppler US in three neonates (fig 2 ). In

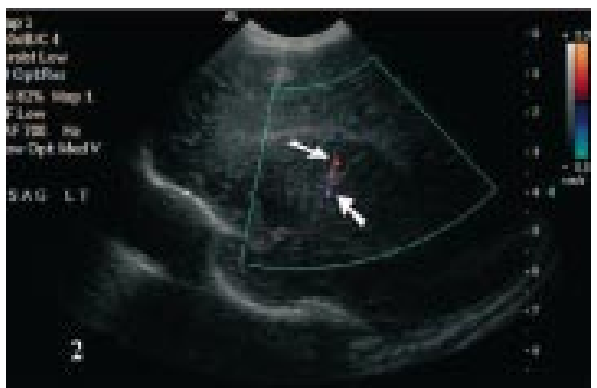

Figure 2 Sagittal colour Doppler image showing the vascular nature of these thalamic echogenicities (arrows).

two of the 21 cases, LSV was first seen on the second US examination at 10 and 21 days of age. LSV was bilateral in 10 of the $21(48 \%)$ cases, and was located in the thalamus in 14 cases and in the basal ganglia in seven. It was accompanied by intraventricular haemorrhage in five cases, and by ventriculomegaly in two.

\section{Comparison of study and control groups}

Table 2 shows a comparison of the study and control groups with regard to various maternal, gestational, and perinatal variables. Compared with the control group, the LSV group included significantly more multiple births $(p=0.013)$, more disturbances in amniotic fluid volume (oligohydramnion or polyhydramnion; $p=0.015)$, and less meconium stained amniotic fluid $(\mathrm{p}<0.01)$. All the remaining variables were not significantly different between the groups. The LSV group included five infants with congenital anomalies: two with cleft lip and palate; one with persistent omphalomesenteric duct; one with congenital contractural arachnodactyly; one with preauricular tags.

Table 3 shows a comparison of the study and control groups with regard to various neonatal variables, medical interventions, and outcome. Compared with the control group, the LSV group required significantly fewer blood transfusions $(p<0.05)$ and less phototherapy $(p<0.01)$. All the remaining variables were not significantly different between the groups. There were no cases of hypothyroidism or twin to twin transfusion.

\section{DISCUSSION}

Our results indicate that LSV is not a rare finding during routine brain US examination of NICU neonates. We encountered LSV in $2.45 \%$ of our NICU admissions, conforming with a reported incidence of $1.9-5.8 \% .^{2-6}$ In our series, $47.6 \%$ of LSV was bilateral, similar to a reported $40-82 \%$ bilateral location. ${ }^{37}$ The incidence of LSV in our series was similar to that previously reported, in spite of including relatively more mature neonates (66.6\% were more than 33 weeks gestation), compared with those described by Chamnanvanakij et al. ${ }^{7}$ Of note is the fact that in only $72.4 \%$ of our NICU admissions was
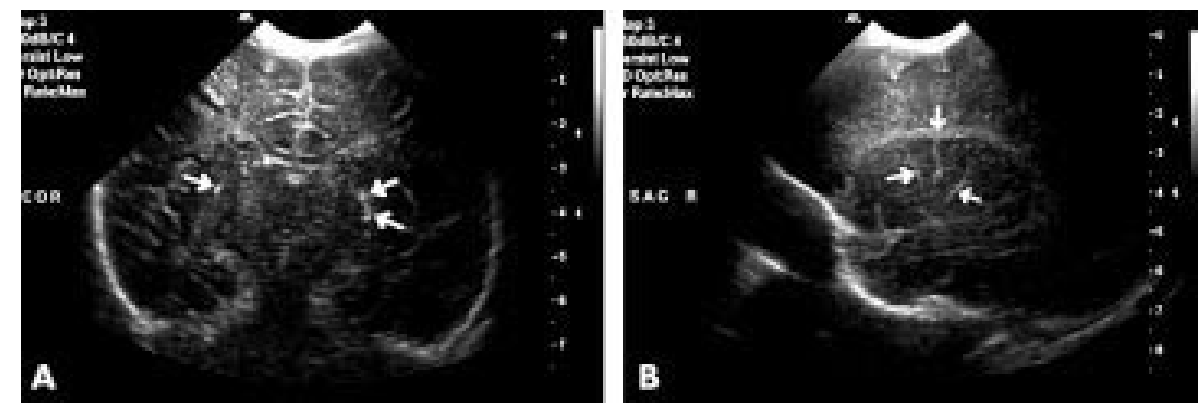

Figure 1 Ultrasound through anterior fontanelle showing branching echogenic linear structures in the thalamus (arrows). (A) Coronal scan; (B) sagittal scan. 
Table 2 Maternal, gestational, and perinatal characteristics of the study and control groups

\begin{tabular}{lll}
\hline Variable & $\begin{array}{l}\text { Study group } \\
(\mathrm{n}=21)\end{array}$ & $\begin{array}{l}\text { Control group } \\
(\mathrm{n}=42)\end{array}$ \\
\hline Mother's age (years) & $27.8(6.7)$ & $30.6(5.4)$ \\
Jewish ethnic origin & $14(66.6 \%)$ & $29(69 \%)$ \\
Spontaneous fertilisation & $16(76.2 \%)$ & $33(78.6 \%)$ \\
Multiple births & $11(52.3 \%)$ & $9(21.4 \%)^{*}$ \\
Maternal smoking in pregnancy & 0 & $3(7.1 \%)$ \\
Maternal drugs during pregnancy & & \\
MgSO & $2(9.5 \%)$ & $1(2.4 \%)$ \\
Indomethacin & $1(4.8 \%)$ & $1(2.4 \%)$ \\
Ritodrine & 0 & $1(2.4 \%)$ \\
Betamethasone & $2(9.5 \%)$ & $7(16.6 \%)$ \\
Gestational problems & & \\
Vaginal bleeding & $2(9.5 \%)$ & $4(9.5 \%)$ \\
PET/PIH/CHT & $2(9.5 \%)$ & $5(11.9 \%)$ \\
GDM/IDDM & 0 & $3(7.1 \%)$ \\
Poly/oligohydramnion & $3 / 3$ & $1 / 1 *$ \\
Alcohol/drug consumption & 0 & 0 \\
Gestational age (weeks) & $35.0(3.3)$ & $35.1(3.4)$ \\
Birth weight (g) & $2352(752)$ & $2388(768)$ \\
Male & $12(57.1 \%)$ & $26(61.9 \%)$ \\
Non-reassuring fetal heart rate & $1(4.8 \%)$ & 0 \\
PROM >12 hours & $3(14.3 \%)$ & $12(28.5 \%)$ \\
Chorioamnionitis & 0 & $3(7.1 \%)$ \\
Meconial amniotic fluids & $1(4.8 \%)$ & $15(35.7 \%)^{*}$ \\
Mode of delivery & $10(47.6 \%)$ & $21(50 \%)$ \\
Vaginal & $10(47.6 \%)$ & $19(45.2 \%)$ \\
Caesarean & 0 & $2(4.8 \%)$ \\
Vacuum/forceps & $9.5 \pm 0.6$ & $9.3 \pm 1$ \\
Five minute Apgar score & $3(14.3 \%)$ & $7(16.7 \%)$ \\
Small for gestational age & $1(4.8 \%)$ & $4(9.6 \%)$ \\
Microcephaly & $5(23.8 \%)$ & $3(7.1 \%)$ \\
Congenital malformations & 0 & $1(2.4 \%)$ \\
Chromosomal aberrations & & \\
\hline & & \\
\hline
\end{tabular}

If not indicated otherwise, values are mean (SD)

* Significantly different from study group.

PET, Pre-eclamptic toxaemia; PIH, pregnancy induced hypertension; CHT, chronic hypertension; GDM, gestational diabetes mellitus; IDDM, insulin dependent diabetes mellitus; PROM, prolonged rupture of membranes.

head US examination carried out, with those not imaged being mostly healthier than those examined. When we matched our LSV cases with controls of the same gestational age, no trends of LSV with gestational age emerged. Therefore we could not determine the incidence of LSV in the normal term neonatal population. However, the gestational age distribution of our LSV cases shows that $28 \%(6 / 21)$ were term infants, whereas only $5 \%(1 / 21)$ were less than 30 weeks gestation.

Routine ultrasound examination of the brain in NICU neonates appears to be the best tool for detecting LSV; vessel patency can be confirmed by colour Doppler US examination..$^{3-5} 8{ }^{11}$ In contrast with US, computed tomography of the brain generally failed to show increased attenuation of vessels in the thalamus or basal ganglia in LSV cases. Brain computed tomography in 60 sonographically diagnosed LSV cases failed to display the lesion, ${ }^{13-68911}$ except in five cases (two with cytomegalovirus; one with toxoplasmosis; one with AIDS; one with fetal alcohol syndrome). ${ }^{6}$ Magnetic resonance imaging also failed to show LSV in most patients studied, ${ }^{3-5}$ and in only four of 23 patients did it show areas of linear increased signal in regions corresponding to the thalamus and basal ganglia. ${ }^{4}$ The superiority of US in detecting LSV is further emphasised by its ability to detect LSV prenatally, as shown by Estroff $e t$ al $^{24}$ in the brain of a fetus with cytomegalovirus.

LSV has been proposed as a marker of diffuse insult to the fetal and neonatal brain. ${ }^{425}{ }^{26}$ Various underlying perinatal and neonatal conditions have been reported to be associated with LSV, mainly fetal TORCH (toxoplasma, other viruses, rubella, cytomegalovirus, herpes virus) infections, ${ }^{1-7} 9$ chromosomal
Table 3 Neonatal morbidities, medical interventions, and outcome in the study and control groups

\begin{tabular}{|c|c|c|}
\hline Variable & $\begin{array}{l}\text { Study group } \\
(n=21)\end{array}$ & $\begin{array}{l}\text { Control group } \\
(n=42)\end{array}$ \\
\hline Respiratory distress syndrome & $8(38 \%)$ & $13(30.9 \%)$ \\
\hline Patent ductus arteriosus & $1(4.8 \%)$ & $1(2.4 \%)$ \\
\hline Apnoea & $1(4.8 \%)$ & $7(16.7 \%)$ \\
\hline Metabolic acidosis $(\mathrm{pH}<7.25)$ & $3(14.3 \%)$ & $3(7.1 \%)$ \\
\hline $\begin{array}{l}\text { Hypoglycaemia (serum glucose } \\
<45 \mathrm{mg} / \mathrm{dl} \text { ) }\end{array}$ & $5(23.8 \%)$ & $18(42.9 \%)$ \\
\hline \multicolumn{3}{|l|}{ Haematological } \\
\hline Haemoglobin $<14 \mathrm{~g} / \mathrm{dl}$ & $7(33.3 \%)$ & $20(47.6 \%)$ \\
\hline Packed cell volume $>65 \%$ & 0 & $1(2.4 \%)$ \\
\hline Leucocytes $<5000 / \mathrm{mm}^{3}$ & 0 & $4(9.6 \%)$ \\
\hline Platelets $<100000 / \mathrm{mm}^{3}$ & $3(14.3 \%)$ & $2(4.8 \%)$ \\
\hline \multicolumn{3}{|l|}{ Hyperbilirubinaemia } \\
\hline Serum bilirubin $>13 \mathrm{mg} / \mathrm{dl}$ & $6(28.6 \%)$ & $18(42.9 \%)$ \\
\hline Peak serum bilirubin (mg/dl) & $11(2.2)$ & $12.2(3.4)$ \\
\hline Congenital viral infection & 1 (CMV) & 0 \\
\hline Acquired bacterial sepsis & $3(14.3 \%)$ & $6(14.3 \%)$ \\
\hline Acquired fungal sepsis & $1(4.8 \%)$ & $1(4.8 \%)$ \\
\hline Necrotising enterocolitis & 0 & 0 \\
\hline \multicolumn{3}{|l|}{ Intraventricular haemorrhage } \\
\hline Grade 1-2 & $4(19 \%)$ & $6(14.3 \%)$ \\
\hline Grade 3-4 & $1(4.8 \%)$ & $2(4.8 \%)$ \\
\hline Seizures & 0 & $2(4.8 \%)$ \\
\hline Mechanical ventilation & $7(33.3 \%)$ & $13(30.9 \%)$ \\
\hline Duration (days) & $2.1(5.2)$ & $1.1(2.1)$ \\
\hline Umbilical artery catheterisation & $3(14.3 \%)$ & $3(7.1 \%)$ \\
\hline Duration (days) & $0.48(1.6)$ & $0.33(1.4)$ \\
\hline Umbilical vein catheterisation & $6(28.6 \%)$ & $10(23.8 \%)$ \\
\hline Duration (days) & $1.52(2.9)$ & $0.83(1.8)$ \\
\hline Surfactant replacement therapy & $4(19 \%)$ & $4(9.6 \%)$ \\
\hline Blood transfusion & $1(4.8 \%)$ & $11(26.2 \%)^{*}$ \\
\hline Exchange transfusion & $1(4.8 \%)$ & $1(2.4 \%)$ \\
\hline Phototherapy & $5(23.8 \%)$ & $25(59.5 \%)$ * \\
\hline Duration (days) & $0.95(1.9)$ & $1.98(2.3)$ \\
\hline Hospital stay (days) & $39.8(81.1)$ & $23.8(17.3)$ \\
\hline Mortality & 0 & 0 \\
\hline \multicolumn{3}{|c|}{$\begin{array}{l}\text { If not indicated otherwise, values are mean (SD). } \\
{ }^{*} \text { Significantly different from study group. } \\
\text { CMV, Cytomegalovirus. }\end{array}$} \\
\hline
\end{tabular}

aberrations, ${ }^{2-689}$ congenital malformations, ${ }^{1568}$ congenital heart disease, ${ }^{4}$ and asphyxia. ${ }^{4}$ Our LSV group was not significantly different from the control group in most variables tested, in spite of including significantly more multiple births. Overall, the neonates with LSV in our study were not sicker than controls, but were actually healthier, with less meconial amniotic fluid, fewer blood transfusions, and less phototherapy. We do not have a sound explanation for the increased incidence of LSV in twins and with abnormal liquor volumes. One speculation is that there is a higher risk of impaired placental flow in these two conditions. Similarly, we could not explain the lower incidence of LSV in infants with meconium stained fluids, or the fact that infants with LSV seem to be less sick. This, however, indicates that LSV did not pose any additional risk in the early neonatal period. Unfortunately, we do not perform routine head US examinations on all infants discharged from the NICU during follow up and therefore we may have missed cases of later LSV.

Table 4 summarises the largest published reports of LSV, including the data from this study. We could not perform a meta-analysis because most of the reports were either retrospective or included a review of brain sonograms without direct reference to patients. In addition, these reports included heterogeneous populations of neonates: premature infants weighing $<1250 \mathrm{~g}^{7}$ NICU admissions, ${ }^{25}$ all neonatal admissions, ${ }^{1}$ and also infants up to 11 months of age. ${ }^{3}$ A summary of LSV cases from large reports with study populations of known size (table 4) shows that, compared with the general neonatal population, patients with LSV have a higher incidence of asphyxia, respiratory disease, congenital heart disease, fetal TORCH infection, chromosomal aberrations, and 


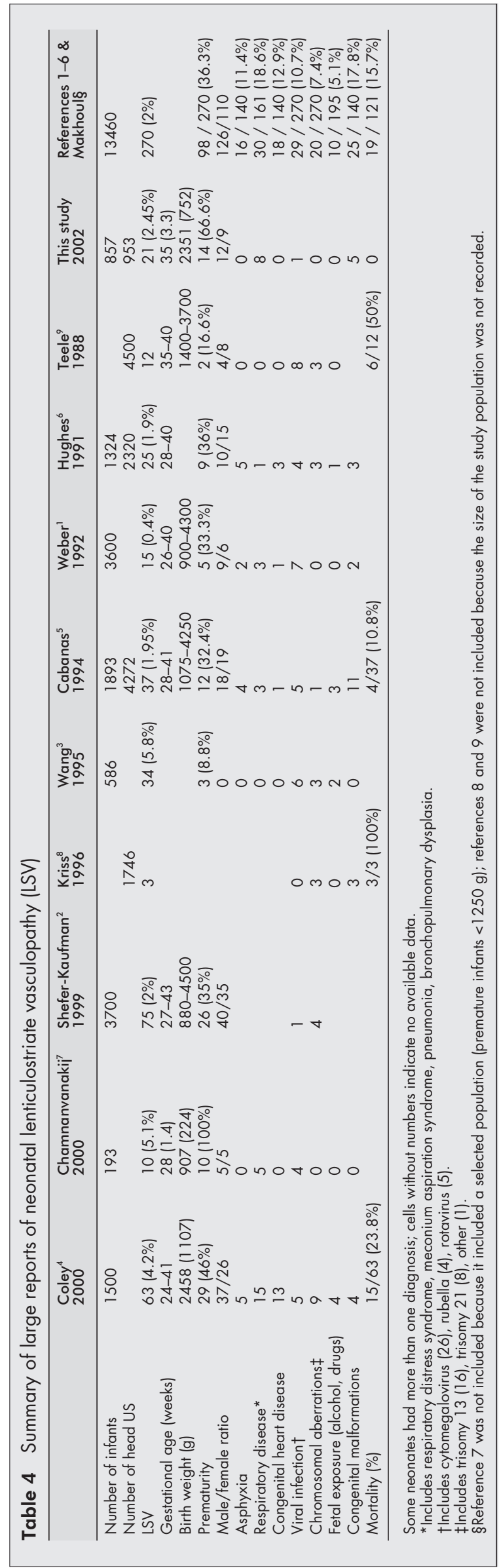

congenital malformations. These findings imply that LSV is most probably associated with these adverse conditions.

Sonographic follow up of patients with LSV up to 15 months of age showed progression of LSV in 14.7-85\% of cases, ${ }^{34}$ no change in $15-86.5 \%,{ }^{135}$ or resolution of lesions in $36.4-50 \%$ of cases. ${ }^{17}$ However, the extent of LSV progression was determined sonographically, but was without clear criteria for the estimation of progression and was operator dependent in terms of awareness of this finding. Nonetheless, progression of LSV could reflect the consequences of brain insult by unfavourable underlying conditions.

Follow up studies reported a neurodevelopmental delay in $18.9-55 \%$ of patients with LSV. ${ }^{15}{ }^{11}$ Furthermore, in premature infants of birth weight $<1250 \mathrm{~g}$, those with LSV had lower scores for mental development, motor quality, and emotional regulation than matched controls. ${ }^{7}$ Most infants with LSV and developmental delay experienced severe disease states, such as cytomegalovirus infection, ${ }^{91}$ major malformations or chromosomal aberrations, ${ }^{15}$ fetal exposure to alcohol, ${ }^{5}$ and systemic Streptococcus sepsis. ${ }^{11}$ Prematurity by itself, with or without respiratory distress syndrome, does not appear to adversely affect the short term developmental outcome of patients with LSV. ${ }^{34}$ In the absence of severe underlying conditions such as fetal TORCH infections, chromosomopathy, major malformations, or hypoxic-ischaemic states, ${ }^{25}{ }^{26}$ survival without adverse clinical outcome is the rule in patients with LSV. However, in survivors with LSV who did not have known associated factors, an initially normal development did not ensure a subsequent favourable course, as long term follow up has shown that some patients develop tics, attention deficit, or hyperactivity disorders. ${ }^{27}$ However, this needs to be confirmed.

The reported mortality in patients with LSV is $8.1-50 \% .^{459}$ Review of the literature shows that those who died had severe underlying conditions, such as trisomy $13,{ }^{49}$ TORCH infections, ${ }^{56}$ major congenital heart diseases, ${ }^{46}$ fetal heroin exposure. $^{5}$ In our series, the mere presence of LSV did not increase mortality and there were no deaths in either the study or control groups.

We conclude that patients with LSV do not have more adverse findings than their matched controls with regard to most gestational, perinatal, and neonatal characteristics and outcome, except for an increased proportion of multiple births. LSV appears to represent a perinatal diffuse insult to the fetal and neonatal brain. A poor outcome in a patient with LSV can be expected when a severe underlying condition coexists. In the absence of known associated conditions, LSV has a good short term prognosis, but unfavourable behavioural and neurological features may become apparent in childhood. Therefore neonatal LSV mandates close neurodevelopmental follow up beyond infancy.

\section{ACKNOWLEDGEMENTS}

We thank Professor Moshe Berant for help and advice in the preparation of the manuscript.

\section{Authors' affiliations}

I R Makhoul, I Eisenstein, P Sujov, T Smolkin, Department of Neonatology, Rambam Medical Center and Rappaport Faculty of Medicine, Technion-Israel Institute of Technology, Haifa, Israel

M Soudack, M Epelman, Department of Radiology, Rambam Medical Center and Rappaport Faculty of Medicine

A Tamir, Department of Community Medicine and Epidemiology, Rambam Medical Center and Rappaport Faculty of Medicine

\section{REFERENCES}

1 Weber K, Riebel T, Nasir R. Hyperechoic lesions in the basal ganglia: an incidental sonographic finding in neonates and infants. Pediatr Radiol 1992;22:182-6.

2 Shefer-Kaufman N, Mimouni FB, Stavorovsky Z, et al. Incidence and clinical significance of echogenic vasculature in the basal ganglia of newborns. Am J Perinatol 1999;16:315-19. 
3 Wang HS, Kuo MF, Chang TC. Sonographic lenticulostriate vasculopathy in infants: some associations and a hypothesis. Am J Neuroradiol 1995;16:97-102.

4 Coley BD, Rusin JA, Boue DR. Importance of hypoxic/ischemic conditions in the development of cerebral lenticulostriate vasculopathy. Pediatr Radiol 2000;30:846-55.

5 Cabanas F, Pellicer A, Morales C, et al. New pattern of hyperechogenicity in thalamus and basal ganglia studied by color Doppler flow imaging. Pediatr Neurol 1994;10:109-16.

6 Hughes $P$, Weinberger $E$, Shaw DW. Linear areas of echogenicity in the thalami and basal ganglia of neonates: an expanded association. Work in progress. Radiology 1991;179:103-5.

7 Chamnanvanakii S, Rogers CG, Luppino C, et al. Linear hyperechogenicity within the basal ganglia and thalamus of preterm infants. Pediatr Neurol 2000;23:129-33.

8 Kriss VM, Kriss TC. Doppler sonographic confirmation of thalamic and basal ganglia vasculopathy in three infants with trisomy 13. J Ultrasound Med 1996:15:523-6.

9 Teele RL, Hernanz-Schulman M, Sotrel A. Echogenic vasculature in the basal ganglia of neonates: a sonographic sign of vasculopathy. Radiology 1988;169:423-7.

10 Grant EG, Williams AL, Schellinger D, et al. Intracranial calcification in the infant and neonate: evaluation by sonography and CT. Radiology 1985; 157:63-8

11 Ben-Ami T, Yousefzadeh D, Backus M, et al. Lenticulostriate vasculopathy in infants with infections of the central nervous system: sonographic and Doppler findings. Pediatr Radiol 1990;20:575-9.

12 Toma $\mathbf{P}$, Magnano GM, Mezzano $P$, et al. Cerebral ultrasound images in prenatal cytomegalovirus infection. Neuroradiology 1989;31:278-9.

13 Chang YC, Huang CC, Liu CC. Frequency of linear hyperechogenicity over the basal ganglia in young infants with congenital rubella syndrome. Clin Infect Dis 1996;22:569-71.

14 Bode H, Rudin C. Calcifying arteriopathy in the basal ganglia in human immunodeficiency virus infection. Pediatr Radiol 1995;25:72-3.
15 Yamashita $Y$, Matsuishi T, Murakami $Y$, et al. Neuroimaging findings (ultrasonography, CT, MRI) in 3 infants with congenital rubella syndrome. Pediatr Radiol 1991;21:547-9.

16 Chabra S, Kriss VM, Pauly TH, et al. Neurosonographic diagnosis of thalamic/basal ganglia vasculopathy in trisomy 13: an important diagnostic aid. Am J Med Genet 1997;72:291-3.

17 Herman TE, Siegel M. Neurosonographic abnormalities in chromosomal disorders. Pediatr Radiol 1991;21:398-401.

18 de Vries LS, Beek FJ, Stoutenbeek P. Lenticulostriate vasculopathy in twin-to-twin transfusion syndrome: sonographic and CT findings. Pediatr Radiol 1995;25(suppl 1):S41-2.

19 Ries M, Deeg KH, Wolfel D, et al. Colour Doppler imaging of intracranial vasculopathy in severe infantile sialidosis. Pediatr Radio 1992;22:179-81

20 Cabanas F, Pellicer A, Valverde E, et al. Central nervous system vasculopathy in neonatal lupus erythematosus. Pediatr Neurol 1996;15:124-6.

21 Bode $\mathbf{H}$, Rudin C, Bubl R. Non calcific vasculopathy in the basal ganglia. Klin Padiatr $1991 ; 203: 350-3$

22 Virkala K, Lappalainen M, Vaalanne L, et al. Radiological signs in newborns exposed to primary toxoplasma infection in utero. Pediatr Radiol 1997:27:133-8.

23 Kao S, Abu-Yousef M. Case 10: pediatric. Mineralizing (lenticulostriate) vasculopathy. J Ultrasound Med 2000;19:362.

24 Estroff JA, Parad RB, Teele RL, et al. Echogenic vessels in the fetal thalami and basal ganglia associated with cytomegalovirus infection. J Ultrasound Med 1992;11:686-8.

25 Wang HS. Neurobehavioral outcome of infants with sonographic lenticulostriate vasculopathy. Pediatr Neurol 2001;24:398.

26 Wang HS. Hypoxic/ischemic etiologic factors in the development of infants with sonographic lenticulostriate vasculopathy. Pediatr Radio 2001;31:674.

27 Wang HS, Kuo MF, Huang SC. Clinical observations of sonographic lenticulostriate vasculopathy in infancy [abstract]. J Neuroimaging $1997 ; 7: 258$

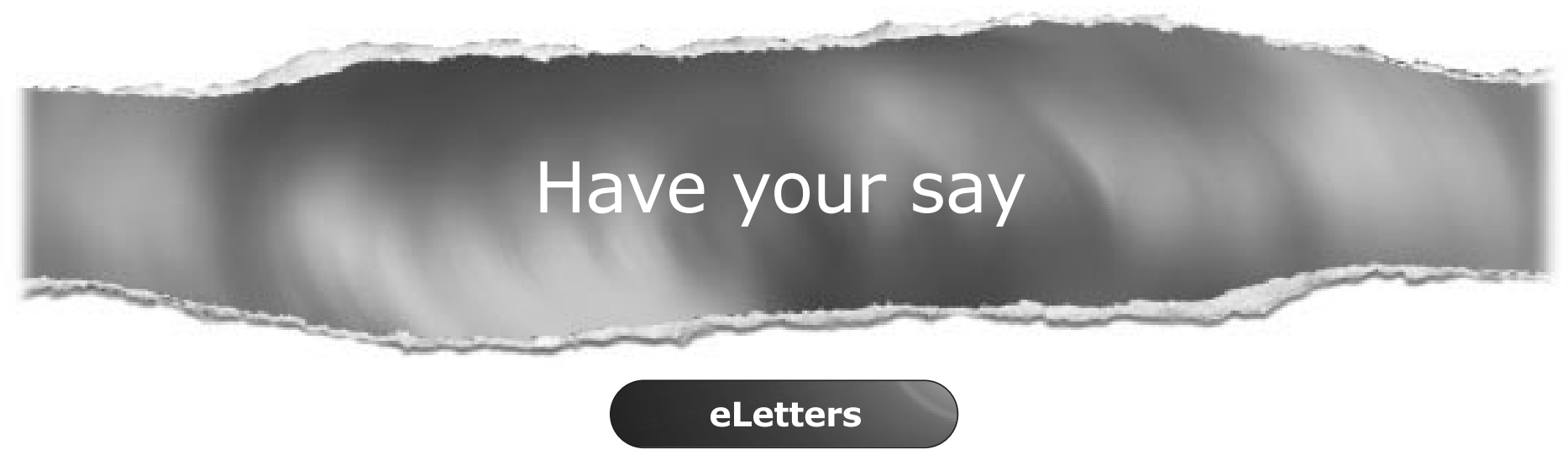

If you wish to comment on any article published in Archives of Disease in Childhood you can send an eLetter using the eLetters link at the beginning of each article. Your response will be posted on Archives of Disease in Childhood online within a few days of receipt (subject to editorial screening).

\section{www.archdischild.com}

